BMJ Open Diabetes

Research \& Care

\title{
Efficacy of intravitreal AFlibercept injection For Improvement of retinal Nonperfusion In diabeTic retinopathY (AFFINITY study)
}

\author{
Yoon Jeon Kim (10 , ${ }^{1}$ Joon Hyung Yeo, ${ }^{2}$ Gisung Son, ${ }^{3}$ Hyojoo Kang, ${ }^{4}$ \\ Yu Sub Sung (D) , ${ }^{5}$ Joo Yong Lee, ${ }^{1}$ June-Gone Kim, ${ }^{1}$ Young Hee Yoon ${ }^{1}$
}

To cite: Kim YJ, Yeo JH, Son G, et al. Efficacy of intravitreal AFlibercept injection For Improvement of retinal Nonperfusion In diabeTic retinopathY (AFFINITY study). BMJ Open Diab Res Care 2020;8:e001616. doi:10.1136/ bmjdrc-2020-001616

- Supplemental material is published online only. To view please visit the journal online (http://dx.doi.org/10.1136/ bmjdrc-2020-001616).

Received 28 May 2020 Revised 15 August 2020 Accepted 19 September 2020

Check for updates

C Author(s) (or their employer(s)) 2020. Re-use permitted under CC BY-NC. No commercial re-use. See rights and permissions. Published by BMJ.

For numbered affiliations see end of article.

Correspondence to Dr Young Hee Yoon; yhyoon@amc.seoul.kr

\section{ABSTRACT}

Introduction To evaluate the effects of intravitreal aflibercept injection on retinal nonperfusion in patients with diabetic retinopathy (DR) using ultrawide field (UWF) fluorescein angiography (FA).

Research design and methods Thirty-eight eyes of 38 consecutive patients with DR and substantial retinal nonperfusion (nonperfusion index (NPI): nonperfused/ total gradable area $>0.2$ ) without macular edema were included in this prospective case series. Monthly injections of $2 \mathrm{mg}$ aflibercept were given for 6 months. UWFfundus photography and UWF-FA images were acquired at baseline, 6 months, and 12 months and evaluated by 2 masked, independent graders for the extent of retinal nonperfusion and vascular leakage. Twenty untreated fellow eyes were analyzed as controls.

Results Inter-grader agreement was strong $(r=0.875)$ for NPI measurements. NPI was $0.46 \pm 0.10$ at baseline; NPI was decreased to $0.43 \pm 0.08(p=0.015)$ after 6 monthly injections of aflibercept and then slightly increased to $0.44 \pm 0.09(p=0.123)$ after 6 months of observation. Vascular leakage also significantly decreased by $21.0 \%$ at 6 months $(p=0.010)$. Untreated fellow eyes did not show significant changes in NPI and vascular leakage during follow-up. Reduction in retinal nonperfusion was associated with severe nonproliferative diabetic retinopathy (NPDR) (vs PDR, OR 19.119, $p=0.025$ ) and higher leakage index (per 0.1, OR 15.152, $p=0.020$ ). Conclusions Intensive aflibercept treatment was effective in reducing retinal capillary nonperfusion in patients with DR without macular edema. Severe NPDR and profound vascular leakage were significantly associated with retinal reperfusion after aflibercept treatment. Trial registration number NCT03006081.

\section{INTRODUCTION}

Retinal ischemia in diabetic retinopathy (DR) leads to vision-threatening complications such as pathological neovascularization, neovascular glaucoma, vitreous hemorrhage, tractional retinal detachments, and macular edema. $^{12}$ Retinal nonperfusion is a useful imaging marker for retinal ischemia ${ }^{3}$ and is known to be associated with the production of vascular endothelial factor (VEGF), which

\section{Significance of this study}

What is already known about this subject?

- Intravitreal anti-vascular endothelial growth factor (VEGF) injection demonstrated a significant improvement in vision with resolution of macular edema and reversed the underlying diabetic retinopathy (DR) severity in patients with diabetic macular edema (DME). There have been controversies regarding the effects of intravitreal anti-VEGF treatment on changes in retinal capillary nonperfusion. Furthermore, the changes of nonperfusion after anti-VEGF injections in patients with sustained retinal nonperfusion without DME is yet to be fully investigated.

What are the new findings?

- In this prospective case series, the nonperfusion index assessed by ultrawide field fluorescein angiography was significantly decreased at 6 months $(p=0.015)$ after 6 monthly injections of aflibercept and then slightly increased $(p=0.123)$ after 6 months of observation. Severe nonproliferative DR (vs proliferative DR) and profound vascular leakage were significantly associated with reduction in retinal nonperfusion after aflibercept treatment.

How might these results change the focus of research or clinical practice?

- Intensive aflibercept treatment was effective in reducing retinal capillary nonperfusion in patients with DR without macular edema. This ability of aflibercept may play a fundamental role in modifying the progress of DR.

is regulated by hypoxia-inducible factor-1 (HIF-1) in hypoxic tissues. ${ }^{4}$ Increased VEGF causes leukostasis, which is presumed to lead to further retinal capillary closure and subsequent exacerbation of retinal ischemia. ${ }^{5-7}$ The advances in ultrawide-field (UWF) retinal imaging technique led to the discovery that the majority of nonperfusion areas (NPAs) in eyes with DR are located outside the posterior 
pole and retinal NPAs are significantly correlated with the DR severity scale (DRSS). ${ }^{89}$

There have been controversies regarding the effects of intravitreal anti-VEGF treatment on changes in retinal nonperfusion. ${ }^{5}{ }^{10-14}$ Several studies reported that neutralization of VEGF reversed the worsening of NPAs in patients with macular edema associated with retinal vein occlusion and DR. ${ }^{5}{ }^{10} 11$ However, as VEGF levels are higher in eyes with macular edema, the presence of macular edema may confound the effects of anti-VEGF treatment on the changes in NPAs. With the increasing use of anti-VEGF therapy for treating advanced DR, the effects of anti-VEGF on the reduction of NPAs in DR without macular edema need to be clarified. We thus carried out a clinical trial to test the efficacy of intravitreal aflibercept injection in reducing retinal nonperfusion in patients with DR without macular edema and identified the factors associated with the changes in retinal nonperfusion.

\section{METHODS}

Study subjects

We performed a prospective, open-label, interventional clinical trial. Informed consent was obtained from each patient prior to enrollment. Patients with DR with significant NPAs (nonperfusion index $(\mathrm{NPI})>0.2$ ) were recruited; NPI was defined as the total area of ischemia divided by the total area of visible retina. Patients were eligible for inclusion if they had type 1 or 2 diabetes, aged 18 years or older, and were willing and able to comply with study-related procedures.

We excluded eyes with coexistent ocular diseases that affected or might affect visual acuity. And we excluded all eyes with diabetic macular edema (DME) showing central retinal thickness (CRT) $\geq 320 \mu \mathrm{m}$ assessed by spectraldomain optical coherence tomography (SD-OCT). Patients with previous treatment with panretinal photocoagulation (PRP) or intraocular surgery other than cataract surgery were also excluded. Other ocular exclusion criteria included media opacity, macular atrophy, fibrovascular proliferation, tractional retinal detachment, and other causes of retinal neovascularization. In addition, patients with elevated intraocular pressure $\geq 22 \mathrm{~mm} \mathrm{Hg}$ despite intraocular pressure-lowering medication or diagnosis of glaucoma in the study eye or best-corrected visual acuity (BCVA) scores of $<34$ letters in the fellow eye were excluded. If both eyes met the criteria, eyes with larger NPI were selected for the study.

Systemic exclusion criteria included HbA1c of $\geq 12 \%$, blood pressure of $\geq 170 / 110 \mathrm{~mm} \mathrm{Hg}$, and presence of renal failure or anticipated need for hemodialysis or peritoneal dialysis during the study.

\section{Study protocol}

All participants visited the clinic every month for 6 months and every 3 months thereafter during a total study period of 12 months and received intravitreal aflibercept injections ( $2 \mathrm{mg} / 0.05 \mathrm{~mL})$ at baseline, 4 weeks, 8 weeks, 12 weeks, 16 weeks, and 20 weeks. BCVA was measured by validated ETDRS visual acuity charts at every 3 months. UWF color fundus photography and SD-OCT images were taken at every visit and UWF-fluorescein angiography (FA) was taken at baseline, 6 months, and 12 months.

Rescue treatments with PRP or intravitreal aflibercept injection were allowed 24 weeks after baseline. PRP was given when there was any sign of proliferative diabetic retinopathy (PDR). Intravitreal aflibercept injection was allowed if study eyes experienced loss of BCVA of $\geq 3$ lines from baseline due to development of DME (CRT of $\geq 320$ $\mu \mathrm{m})$. For the fellow eye, treatment for DME was applied according to the investigator's discretion. Further, the fellow eyes that met the inclusion criteria and did not receive anti-VEGF treatments during the study period were included in the control group for interocular comparisons.

\section{Image acquisition}

UWF-fundus photography and UWF-FA were performed using the Optos California (Optos, Dunfermline, UK). Each image output was automatically corrected for projection errors using the OptosAdvance software (Optos) that uses stereographic projection techniques. The FA images were acquired in the early phase (up to $60 \mathrm{~s}$ ), recirculation phase (2 to $3 \mathrm{~min}$ ), and late phase at 5 to $7 \mathrm{~min}$. Two images were selected from the early phase that most clearly presented the margin of nonperfused territories and from the recirculation phase that differentially presented the degree of vascular leakage, respectively. The images were extracted at a resolution of $300 \times 300$ dpi.

\section{Image analysis}

A novel ImageJ-based (NIH ImageJ; National Institutes of Health, Bethesda, MD, USA) software named "AsanJ-OPH" was developed for the analysis of UWF-FA images by Son et al. ${ }^{15} \mathrm{NPA}$ and vascular leakage were quantitatively analyzed in a semi-automated manner using this software, which showed strong agreement with manually determined NPA and acceptably high intraobserver and interobserver agreements. ${ }^{15}$ In the current study, the images were analyzed using AsanJ-OPH by two trained retinal specialists (JHY and GS) who were blinded to the clinical information, and the averages of the two sets of results were used in the analysis. In case of inconsistency, the values were verified by a third retina specialist $(\mathrm{YJK})$.

For topographical analysis, three concentric circles were drawn over the UWF-FA images (see online supplemental eFigure 1). The foveal center was set as the center of circles and the disc to macular distance was set as the radius of the smallest circle. The radii of the middle and largest circles were set as double and triple of the radius of the smallest circle, respectively. In this way, the smallest, middle, and the largest zone encompassed approximately $30^{\circ}, 60^{\circ}$, and $90^{\circ}$ of the fundus. In 
addition, the entire field was divided into four quadrants centered at the optic disc and designated as superior (S), inferior $(\mathrm{I})$, temporal $(\mathrm{T})$, and nasal $(\mathrm{N})$.

The total visible retina was delineated along the clearly visible margin of the retinal vasculature in the fundus. We unified the extent of total visible retina in each fundus image taken at baseline, 6 months, and 12 months. And to analyze the NPAs, regions of interest (ROIs) of a specified minimum size (default value of $\geq 1000$ pixels) and a luminosity below a certain threshold were selected. Once automatically selected with the software, the area, threshold, and the margin of the ROI could be manually determined by the researcher with the magic wand tool and ROIs identified as non-NPAs were removed. The NPIs of the total, each zone, or quadrant were then calculated by dividing the nonperfusion area by the area of the corresponding zone or quadrant.

Diffuse vascular leakage was quantified as the vascular leakage index. The signal intensities of the total vessels were measured using the luminosity values of major vessels truncated at the optic disc as the reference value. A representative measurement of vascular leakage index is shown in online supplemental eFigure 2.

\section{Statistical analysis}

Descriptive statistics (number and percentage for categorical variables and mean \pm SD for continuous variables) were evaluated in order to determine the baseline characteristics of subjects. The NPIs and vascular leakage at each time point were presented as continuous variables and the changes from baseline were assessed with paired Student's t-test. ORs for factors potentially associated with the improvement of retinal nonperfusion were calculated using binary logistic regression analysis. All statistics were performed with IBM SPSS Statistics for Windows, V.20.0.

\section{RESULTS}

A total of 38 eyes from 38 patients were included in the study and received intravitreal aflibercept. The baseline characteristics of the patients are shown in table 1. The patients had an average age of $54.8 \pm 10.3$ years, HbAlc level of $8.1 \pm 1.4 \%$, and the mean duration of diabetes of $15.5 \pm 5.9$ years. Distribution of DRSS was as follows: moderate nonproliferative DR (NPDR), 4 (10.5\%); severe NPDR, 8 (21.1\%); very severe NPDR, 15 (39.5\%); early PDR, $11(28.9 \%)$. All patients were retained until the final visit (see online supplemental eFigure 3). One study eye underwent rescue PRP after vitreous hemorrhage at 10 months, and four and one fellow eyes underwent PRP and vitrectomy, respectively, for progression of DR. None of the study eyes developed DME during follow-up, and five fellow eyes received anti-VEGF treatment for DME.

Image analysis with AsanJ-OPH software showed high inter-observer agreements between two retinal specialists: the intraclass correlation (ICC) values were 0.846 for total visible retina, 0.835 for NPAs, 0.875 for NPI, and 0.936
Table 1 Baseline characteristics of the study eyes

Total ( $\mathrm{n}=\mathbf{3 8})$

\begin{tabular}{ll}
\hline Demographics & $54.8 \pm 10.3$ \\
\hline Age, years & $11(28.9)$ \\
\hline Women, N (\%) & $23(60.5)$ \\
\hline Hypertension, N (\%) & $13(34.2)$ \\
\hline Hyperlipidemia, N (\%) & $15.5 \pm 5.9$ \\
\hline Duration of diabetes, years & $8.1 \pm 1.4$ \\
\hline HbA1C, \% & \\
\hline Medication & $22(57.9)$ \\
\hline Oral hypoglycemic agents only, N (\%) & $16(42.1)$ \\
\hline Insulin and oral hypoglycemic agents, & \\
\hline N (\%) & \\
\hline Ocular characteristics & $0.10 \pm 0.18(20 / 25)$ \\
\hline BCVA, logMAR (in Snellen) & \\
\hline Diabetic retinopathy severity & $4(10.5)$ \\
\hline Moderate NPDR, N (\%) & $8(21.1)$ \\
\hline Severe NPDR, N (\%) & $15(39.5)$ \\
\hline Very severe NPDR, N (\%) & $11(28.9)$ \\
\hline Early PDR, N (\%) & \\
\hline Lens status & $31(81.6)$ \\
\hline Phakic, N (\%) & $7(18.4)$ \\
\hline Pseudophakic, N (\%) & \\
\hline Previous ocular treatment & $4(10.5)$ \\
\hline Anti-VEGF therapy & $1(2.6)$ \\
\hline Steroid therapy & $-0.67 \pm 1.60$ \\
\hline Refractive error spherical equivalent, D & $16.2 \pm 3.4$ \\
\hline Intraocular pressure, mm Hg & $290.5 \pm 70.0$ \\
\hline Central retinal thickness, $\mu m$ & $252.3 \pm 42.7$ \\
\hline Subfoveal choroidal thickness, $\mu \mathrm{mm}$ & \\
\hline & \\
\hline
\end{tabular}

BCVA, best-corrected visual acuity; NPDR, non-proliferative diabetic retinopathy; PDR, proliferative diabetic retinopathy; VEGF, vascular endothelial growth factor.

for vascular leakage. At baseline, the NPI was $0.46 \pm 0.10$ and the vascular leakage index was $0.28 \pm 0.10$. The ICCs for NPI ranged from 0.855 to 0.944 in topographic analyses. NPIs of superior and inferior quadrants were significantly higher than those of nasal and temporal quadrants $(\mathrm{p}<0.001)$ (see online supplemental eTable 1$)$, and the NPI increased from the posterior pole to the periphery, in the order of zone 1,2 , and 3 .

After 6 monthly injections of aflibercept, the NPI of the total visible retina decreased to $0.43 \pm 0.08(\mathrm{p}=0.015)$; at the last visit after the 6-month observation period, the NPI had slightly increased to $0.44 \pm 0.09(\mathrm{p}=0.123)$ (see online supplemental eTable 1, figures 1 and 2A). Topographically, the changes in NPI after aflibercept injections were noted in the superior and inferior quadrants and zone 3 (figure 2B and C). Vascular leakage significantly decreased by $21.0 \%$ between baseline and 6 months 
(A)

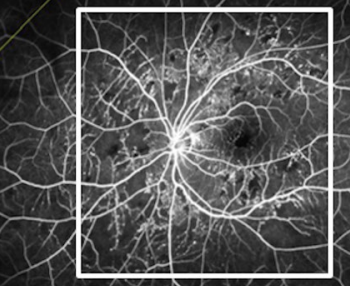

(B)

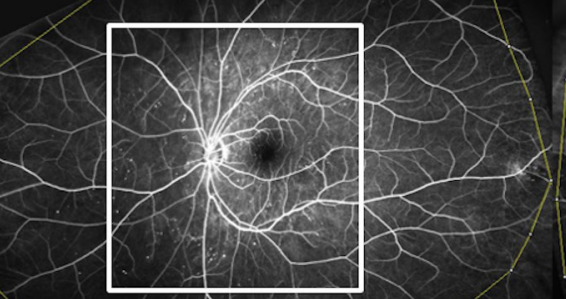

(C)

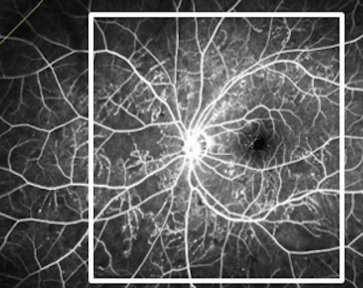

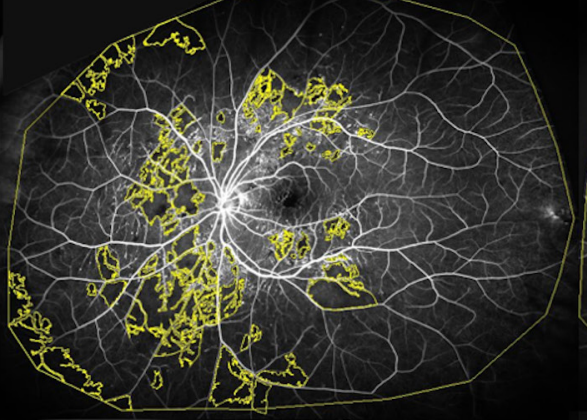
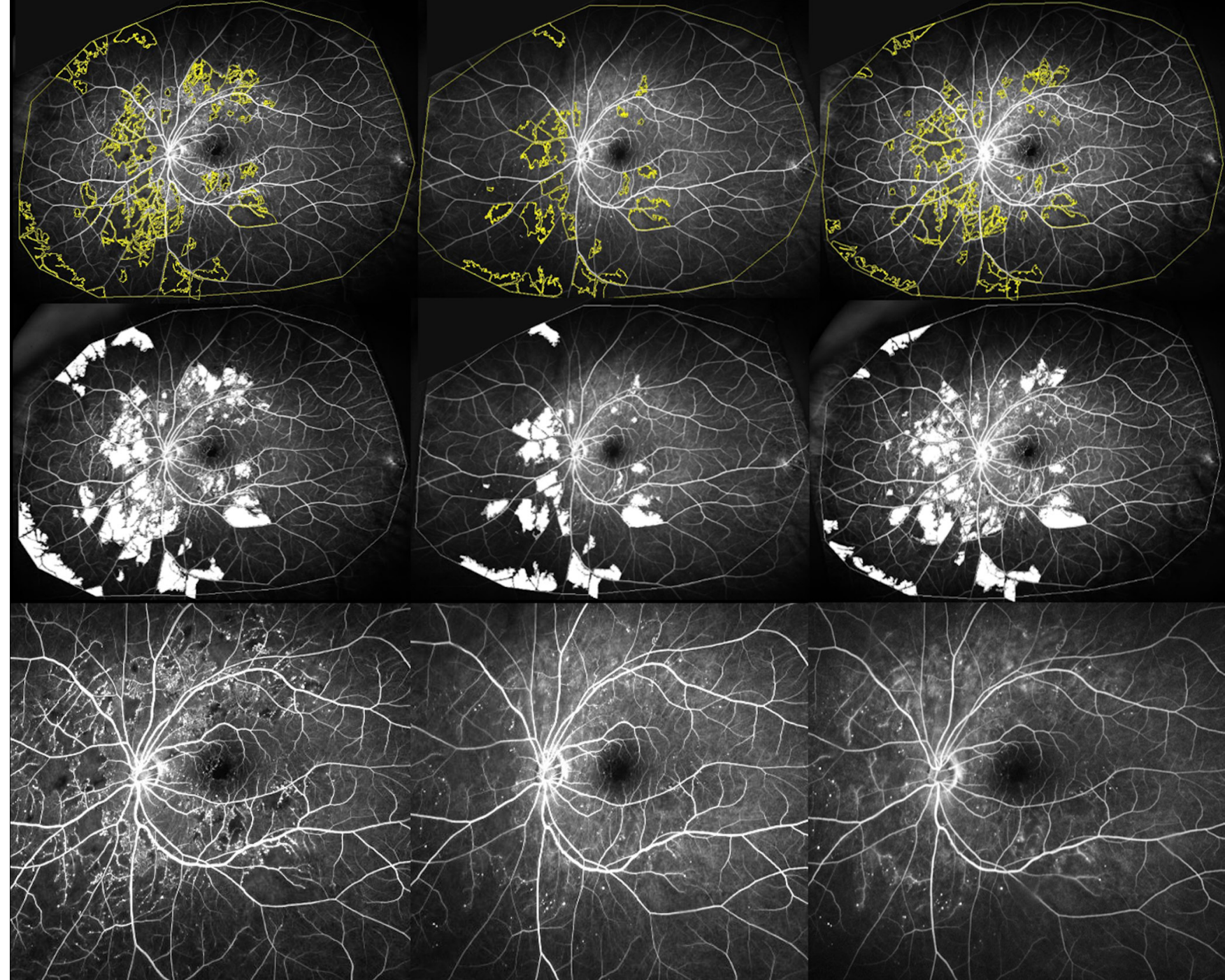

Figure 1 Representative ultrawide field fluorescein angiographic images from a patient with significant improvement in retinal perfusion after aflibercept therapy. (A) Nonperfusion index (NPI) at baseline: 0.21. (B) NPI at 6 months: 0.15. (C) NPI at 12-month visit: 0.17 . The white rectangles show an area of interest enlarged in the lowest row.

(baseline, 0.28 $\pm 0.10 ; 6$ months, $0.22 \pm 0.06 ; \mathrm{p}=0.010$ ) (figure 3, online supplemental eFigure 4). After another 6 months of observation, the vascular leakage index was not significantly different from the baseline (12 months, $0.25 \pm 0.07 ; \mathrm{p}=0.133)$. NPAs and vascular leakage were measured at $55.4 \pm 9.3 \mathrm{~s}$ and $171.1 \pm 23.0 \mathrm{~s}$ after dye injection, respectively, and the time of measurement was not significantly different among the visits $(p>0.05)$.
The DRSS was significantly improved after six consecutive injections of aflibercept $(p=0.025)$ (see online supplemental eFigure 5). However, the difference between baseline and 12 months was not significant after 6 months of observation. While there were no significant changes regarding BCVA (baseline, $0.10 \pm 0.18$; 6 months, $0.07 \pm 0.07 ; 12$ months, $0.11 \pm 0.13$; all $\mathrm{p}>0.05)$, CRT showed similar changes to DRSS during the study period. 
(A)

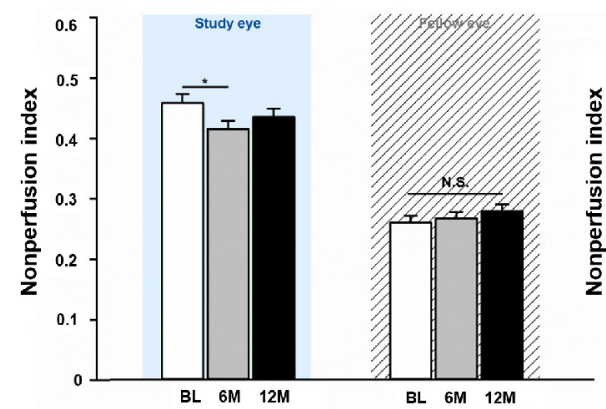

(B)

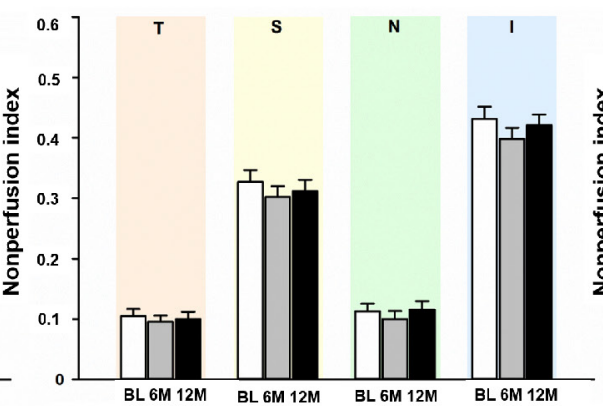

(C)

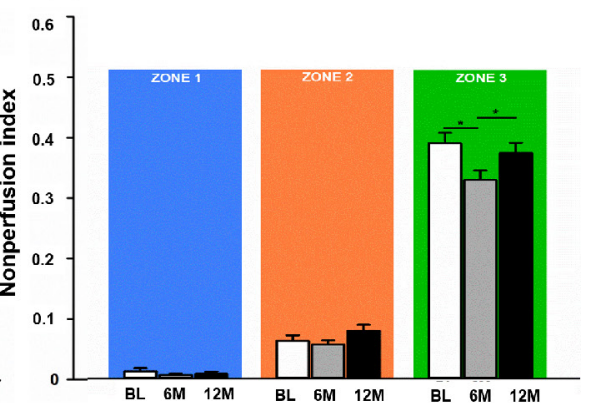

Figure 2 Changes in nonperfusion index (NPI) after 6 monthly aflibercept treatments. (A) NPIs of study eyes and fellow eyes. Topographical analysis of $(B)$ the four quadrants and $(C)$ three concentric zones.

Despite the absence of macular edema at baseline, CRT significantly decreased at 6 months compared with baseline (baseline, 281.9 $434.8 \mu \mathrm{m}$; 6 months, $265.8 \pm 30.4 \mu \mathrm{m}$; $\mathrm{p}=0.001)$. After another 6 months, this decrease became insignificant compared with baseline (12 months, $276.7 \pm 32.0 \mu \mathrm{m} ; \mathrm{p}=0.075$ ).

For interocular comparisons, a total of 20 patients whose fellow eyes met the inclusion criteria and did not receive treatments during the study period were analyzed as controls. Unlike the study eyes, fellow eyes did not show significant changes in the NPI and vascular leakage during follow-up (all $\mathrm{p}>0.05$ ) (figures $2 \mathrm{~A}$ and 3, online supplemental eFigure 4). In addition, the CRT of fellow eyes did not show significant changes throughout the entire study period (baseline, 282.0 $\pm 34.2 \mu \mathrm{m} ; 6$ months, 282.7 $\pm 32.2 \mu \mathrm{m} ; 12$ months, $282.6 \pm 35.9 \mu \mathrm{m}$; all $\mathrm{p}>0.05$ ).

Table 2 shows the univariate and multivariate ORs for each putative factor associated with the reduction of retinal NPAs. In multivariate analysis, improvement in retinal nonperfusion after aflibercept injection was associated with severe NPDR (vs PDR, OR 19.119, p=0.025) and higher vascular leakage index (per 0.1, OR 15.152, $\mathrm{p}=0.020$ ). The changes in retinal nonperfusion in terms of baseline DRSS are shown in online supplemental eFigure 6-specifically, eyes with severe NPDR showed

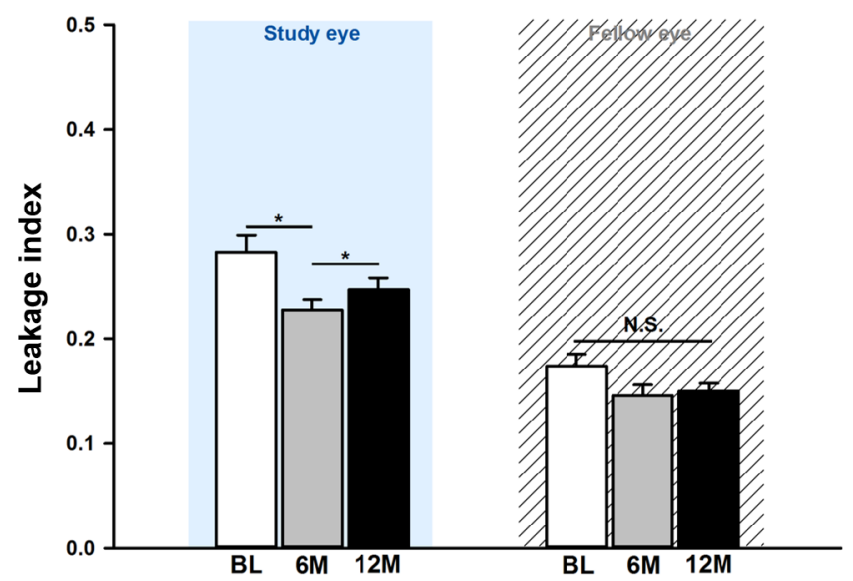

Figure 3 Changes in vascular leakage index after six monthly aflibercept treatments. the most prominent improvement in retinal nonperfusion following aflibercept treatment.

\section{DISCUSSION}

Our results demonstrated that the total area of retinal capillary nonperfusion was significantly reduced after 6 monthly aflibercept injections in patients with substantial NPAs without DME. These data suggest that by increasing retinal perfusion with the blockade of VEGF, the anti-VEGF treatment could suppress the fundamental pathophysiology of DR and play a role in modifying the progress of DR. Such improvements were especially prominent in eyes with severe NPDR and severe vascular leakage at baseline. In contrast, fellow eyes did not show significant improvements in NPI and vascular leakage.

Several previous studies noted that aflibercept improves retinal perfusion, ${ }^{5} 1011$ which is in line with our study. However, these studies mostly focused on the posterior pole of the fundus when assessing retinal nonperfusion. In this study, we used UWF retinal imaging to detect pathology in the periphery, where the NPAs most frequently develop but may be missed on conventional FA images. ${ }^{9}{ }^{16-18}$ As a result, we found that improvement in perfusion following anti-VEGF therapy most prominently occurred outside the $60^{\circ}$ range in the superior and inferior quadrants. Similar to the results of previous studies, the improvement in retinal perfusion of the posterior pole was noted in our study (as demonstrated in figure 2), although there was no significant difference probably due to the differences in patient population and study protocols. While the previous studies included patients with DME who had poorer macular perfusion, our study participants did not have DME and showed relatively preserved macular perfusion at baseline.

The effect of anti-VEGF on the improvement of retinal nonperfusion observed in our study did not last up to 1 year after 6 months of discontinuation, meaning that the initial improvement observed in our study reflected the actual therapeutic effect of anti-VEGF and was not by chance. In terms of cost-effectiveness, the use of antiVEGF injections for reducing NPA in the eyes with DR 
Table 2 Factors associated with improvement in retinal nonperfusion after intravitreal aflibercept injections

\begin{tabular}{|c|c|c|c|c|}
\hline & Univarie & & Multiva & \\
\hline & OR & $P$ value & OR & $P$ value \\
\hline Demographics & & & & \\
\hline Age, years & 0.921 & 0.138 & & \\
\hline Female sex & 0.525 & 0.417 & & \\
\hline Hypertension & 0.292 & 0.092 & & \\
\hline Duration of diabetes, years & 0.938 & 0.355 & & \\
\hline $\mathrm{HbA1C}, \%$ & 1.213 & 0.474 & & \\
\hline Insulin and $\mathrm{OHA}, \mathrm{N}$ (vs OHA only) & 2.602 & 0.955 & & \\
\hline Ocular characteristics & & & & \\
\hline BCVA, logMAR & 1.618 & 0.832 & & \\
\hline Diabetic retinopathy severity (vs early & & & & \\
\hline Moderate NPDR & 18.000 & 0.074 & 20.904 & 0.071 \\
\hline Severe NPDR & 15.000 & 0.035 & 19.119 & 0.025 \\
\hline Very severe NPDR & 5.000 & 0.177 & 3.244 & 0.083 \\
\hline Pseudophakic lens status & 1.021 & 0.789 & & \\
\hline Refractive error, D & 1.413 & 0.525 & & \\
\hline Intraocular pressure, $\mathrm{mm} \mathrm{Hg}$ & 0.650 & 0.822 & & \\
\hline Presence of PPL & 0.521 & 0.360 & & \\
\hline Nonperfusion index (per 0.1) & 1.111 & 0.031 & & \\
\hline Leakage index (per 0.1) & 12.063 & 0.005 & 15.152 & 0.020 \\
\hline Central retinal thickness, $\mu \mathrm{m}$ & 1.955 & 0.652 & & \\
\hline Subfoveal choroidal thickness, $\mu \mathrm{m}$ & 1.770 & 0.240 & & \\
\hline
\end{tabular}

BCVA, best-corrected visual acuity; NPDR, nonproliferative diabetic retinopathy; OHA, oral hypoglycemic agents; PDR, proliferative diabetic retinopathy; PPL, predominantly peripheral lesion.

without macular edema would have limited value. In addition, since the anti-VEGF treatment alone cannot sufficiently improve a wide range of nonperfusion, replacing PRP with the anti-VEGF treatment alone is impossible for the treatment of DR. However, in cases that nonperfusion progresses rapidly or progresses to the posterior pole, the anti-VEGF treatment, when used as an auxiliary treatment to PRP, could be useful for preserving the posterior pole and delaying the timing of PRP or reducing the range of PRP as much as possible. Moreover, when the sustained delivery of VEGF antagonists becomes possible, this purpose of anti-VEGF use would become more feasible.

The underlying mechanism for improvement in retinal perfusion by intensive anti-VEGF treatment is yet to be fully elucidated. A possible explanation is that anti-VEGF treatment relieves leukostasis caused by VEGF secreted in the ischemic environment, thereby preventing further retinal capillary closure and subsequent exacerbation of retinal ischemia. ${ }^{5-7}$ There might be a portion of circulation that is closed but not permanently, and this reversible closure may likely be modulated by VEGF. We found that patients with severe NPDR experienced the most significant reduction of nonperfusion among the study groups-considering this, the reversal of capillary perfusion seems to occur in eyes in a certain range of diabetic changes before the development of irreversible capillary damage.

Another possible explanation of the therapeutic effect of anti-VEGF treatment is the increase in the stability of vascular walls and subsequent improvement of overall efficiency in retinal perfusion. Anti-VEGF agents reduce vascular permeability by restoring pericytes and normalizing the Bruch's membrane. ${ }^{19}$ This is supported by our findings in which patients with severe vascular leakage showed a significant improvement in retinal perfusion, along with decreases in vascular leakage. Moreover, in NPAs with vascular leakage, there may be viable tissue that can produce VEGF and other cytokines that cause vascular leakage. ${ }^{20}$ Therefore, taken together, we suggest that delayed perfusion improves in the viable retina with reversibility in hypoperfusion or delayed perfusion before permanent closure.

In our topographical analysis, the changes of NPAs after aflibercept injections were most prominent in the superior and peripheral regions, where the baseline NPIs were relatively larger. In addition, although the number of patients was small, those with moderate NPDR did not show significant improvements in retinal nonperfusion. Similar to the 
results of previous studies in which eyes with more severe diseases had more favorable VA and anatomic outcomes after aflibercept treatment, ${ }^{21}{ }^{22}$ our findings may have been affected by the "ceiling effect" in which smaller NPA at baseline leads to less room for improvement. On the other hand, as aforementioned, we found that the degree of improvement was smaller in patients in whom the DR had progressed to a more severe form (ie, PDR) with irreversible capillary closure than in those with less severe diseases. Based on these findings, we suggest that DRSS and NPAs at baseline may be the characteristics potentially associated with improvement in retinal nonperfusion.

It is possible that changes in the retinal thickness and vascular leakage with the anti-VEGF treatment affected the interpretation of the nonperfusion. However, to diminish the effect of retinal thickness on the measurement of nonperfusion, we did not include eyes with DME in the current study. Since eyes with significant DME were not included in our study, visualization of vessels might not have differed depending on the change in retinal thickness during the study period. As for the leakage, we adopted the images taken between $30 \mathrm{~s}$ and $1 \mathrm{~min}$ to preclude the effect of leakage noted in the late phases of FA in the measurement of NPAs. In addition, we set the absolute signal intensity to delineate the nonperfusion area and did not change the FA settings during the whole study period.

The functional recovery by the reversal of NPA with delayed perfused vessels to perfused vessels with slow velocities and its clinical importance remain to be clarified. The reversal of retinal perfusion that recanalizes the nonperfusion area with delayed perfused vessels to perfused vessels with slow velocities might not correlate with a functional recovery of substantial clinical importance. However, recovery of blood flow with early treatment before retinal function completely declines would be meaningful.

The results of this study are somewhat inconsistent with those of the substudy of the CLARITY trial ${ }^{12} 13$ and the RECOVERY trial. ${ }^{14}$ In the CLARITY study, ${ }^{12}$ the authors did not observe differences in intravascular oxygen saturation or NPAs between the PRP and intravitreal aflibercept treatment groups. Likewise, the RECOVERY study did not reveal a significant impact of intravitreal aflibercept on retinal nonperfusion in eyes with PDR. Such disparity with our current results is likely due to differences in study protocols as well as the characteristics of study subjects. First, we included a smaller portion of active PDR eyes (early PDR, 29\%) than did the RECOVERY trial in which all subjects had PDR. In addition, while we did not include previous history of PRP treatment at baseline, $33 \%$ of subjects in the CLARITY substudy had undergone PRP treatment prior to enrollment. Because PRP confers permanent effects on the amount and property of the NPAs, such difference may have led to disparities in study outcomes. Second, the numbers of aflibercept injections were different between our study and others. The amount of anti-VEGF treatment required for adequate reversal of retinal nonperfusion is yet uncertain.

Recent studies reported that OCT angiography demonstrated that suspected reperfusions in eyes with DR following anti-VEGF treatment were indeed consistently nonperfused. ${ }^{23}$ However, because the OCTA technology only detects vessels with flow rates above a certain velocity, vessels with low flow rates that have resumed blood flow on anti-VEGF therapy cannot be readily visualized using OCT angiography. Furthermore, whereas OCT angiography can only observe posterior parts of the retina, most of the retinal NPAs are located in the peripheral retina, in which the changes in NPAs after anti-VEGF therapy are most commonly observed.

\section{Limitations}

This study has several limitations of note. First, this was a short-term study assessing the efficacy of anti-VEGF injections, so their long-term effects were not evaluated. Considering that the improvement in retinal nonperfusion was not significant at the 1-year follow-up after 6 months of treatment discontinuation, further studies are needed to determine the appropriate monitoring protocol for eyes following discontinuation of antiVEGF treatment. Second, we did not include a control group in the analysis. To compensate for the lack of a control group, we performed inter-ocular comparisons in subjects whose fellow eyes met the inclusion criteria and did not receive treatments during the study period. Third, we used the images taken between $30 \mathrm{~s}$ and 1 min in order to preclude the effect of leakage noted at the late phases of FA in measurement of NPAs. Therefore, our data might reflect the recovery of delayed perfusion other than permanent NPAs, both of which are regarded to be related to tissue hypoxia. Lastly, the single-centered images that we used capture $\sim 80 \%$ of the fundus compared with montaged images, and the pathologic lesions in the far periphery may have been underestimated. ${ }^{16}$ Nevertheless, these limitations are not likely to have significantly affected our findings because the main goal of this study was to perform intra-individual comparisons regarding NPI changes.

\section{CONCLUSIONS}

We report that aflibercept treatment was effective in improving NPAs in eyes with DR without macular edema. These changes were particularly notable in eyes with severe capillary leakage or severe NPDR, and less obvious in eyes with either smaller baseline NPA or PDR with irreversible closure. This ability of aflibercept to improve retinal perfusion may play a fundamental role in modifying the progress of DR.

Author affiliations

${ }^{1}$ Department of Ophthalmology, Asan Medical Center, University of Ulsan College of Medicine, Seoul, Korea 
${ }^{2}$ Department of Ophthalmology, College of Medicine, Chung-Ang University, Seoul, Korea

${ }^{3}$ Department of Retina Service, Hangil Eye Hospital, Incheon, Korea

${ }^{4}$ Diabetic Retinopathy Clinic, Asan Medical Center, Seoul, Korea

${ }^{5}$ Department of Radiology and Research Institute of Radiology, Asan Medical Center, University of Ulsan College of Medicine, Seoul, Korea

Contributors YJK, JYL, J-GK, and YHY were responsible for study concept and design, and drafting and critical revision of the manuscript for important intellectual content. YJK, JHY, GS, HK, YSS, and YHY were responsible for the acquisition, analysis, and interpretation of the data.

Funding This study was supported by Basic Science Research Program through the National Research Foundation of Korea (NRF) funded by the Ministry of Education (2017R1D1A1B05028221) and by Bayer HealthCare.

Disclaimer The funder had no roles in study design, patient recruitment, data collection, data analysis, data interpretation, writing or editing of the report, or the decision to submit for publication.

Competing interests None declared.

Patient consent for publication Not required.

Ethics approval The study was conducted according to the tenets of the Declaration of Helsinki, and all study-related data acquisitions were approved by the Institutional Review Board of Asan Medical Center (Seoul, Korea, IRB No. 2016-0012).

Provenance and peer review Not commissioned; externally peer reviewed.

Data availability statement Data are available on reasonable request.

Supplemental material This content has been supplied by the author(s). It has not been vetted by BMJ Publishing Group Limited (BMJ) and may not have been peer-reviewed. Any opinions or recommendations discussed are solely those of the author(s) and are not endorsed by BMJ. BMJ disclaims all liability and responsibility arising from any reliance placed on the content. Where the content includes any translated material, BMJ does not warrant the accuracy and reliability of the translations (including but not limited to local regulations, clinical guidelines, terminology, drug names and drug dosages), and is not responsible for any error and/or omissions arising from translation and adaptation or otherwise.

Open access This is an open access article distributed in accordance with the Creative Commons Attribution Non Commercial (CC BY-NC 4.0) license, which permits others to distribute, remix, adapt, build upon this work non-commercially, and license their derivative works on different terms, provided the original work is properly cited, appropriate credit is given, any changes made indicated, and the use is non-commercial. See: http://creativecommons.org/licenses/by-nc/4.0/.

ORCID iDs

Yoon Jeon Kim http://orcid.org/0000-0003-4293-9641

Yu Sub Sung http://orcid.org/0000-0002-9215-735X

\section{REFERENCES}

1 Singer M, Tan CS, Bell D, et al. Area of peripheral retinal nonperfusion and treatment response in branch and central retinal vein occlusion. Retina 2014;34:1736-42.

2 Patel RD, Messner LV, Teitelbaum B, et al. Characterization of ischemic index using ultra-widefield fluorescein angiography in patients with focal and diffuse recalcitrant diabetic macular edema. Am J Ophthalmol 2013;155:1038-44.

3 Tsui I, Williams BK, Kok YO, et al. Reliability of ischemic index grading in common retinal vascular diseases. Ophthalmic Surg Lasers Imaging Retina 2015;46:618-25.

4 Hofman P, van Blijswijk BC, Gaillard PJ, et al. Endothelial cell hypertrophy induced by vascular endothelial growth factor in the retina: new insights into the pathogenesis of capillary nonperfusion. Arch Ophthalmol 2001;119:861-6.
5 Campochiaro PA, Wykoff CC, Shapiro H, et al. Neutralization of vascular endothelial growth factor slows progression of retinal nonperfusion in patients with diabetic macular edema. Ophthalmology 2014;121:1783-9.

6 Liu Y, Shen J, Fortmann SD, et al. Reversible retinal vessel closure from VEGF-induced leukocyte plugging. JCI Insight 2017;2:e95530.

7 Tolentino MJ, Miller JW, Gragoudas ES, et al. Intravitreous injections of vascular endothelial growth factor produce retinal ischemia and microangiopathy in an adult primate. Ophthalmology 1996;103:1820-8.

8 Fan W, Wang K, Ghasemi Falavarjani K, et al. Distribution of nonperfusion area on ultra-widefield fluorescein angiography in eyes with diabetic macular edema: DAVE study. Am J Ophthalmol 2017;180:110-6.

9 Silva PS, Dela Cruz AJ, Ledesma MG, et al. Diabetic retinopathy severity and peripheral lesions are associated with nonperfusion on ultrawide field angiography. Ophthalmology 2015;122:2465-72.

10 Campochiaro PA, Bhisitkul RB, Shapiro H, et al. Vascular endothelial growth factor promotes progressive retinal nonperfusion in patients with retinal vein occlusion. Ophthalmology 2013;120:795-802.

11 Wykoff CC, Shah C, Dhoot D, et al. Longitudinal retinal perfusion status in eyes with diabetic macular edema receiving intravitreal aflibercept or laser in VISTA study. Ophthalmology 2019;126:1171-80

12 Nicholson L, Crosby-Nwaobi R, Vasconcelos JC, et al. Mechanistic evaluation of panretinal photocoagulation versus aflibercept in proliferative diabetic retinopathy: CLARITY substudy. Invest Ophthalmol Vis Sci 2018;59:4277-84.

13 Sivaprasad S, Prevost AT, Vasconcelos JC, et al. Clinical efficacy of intravitreal aflibercept versus panretinal photocoagulation for best corrected visual acuity in patients with proliferative diabetic retinopathy at 52 weeks (CLARITY): a multicentre, single-blinded, randomised, controlled, phase $2 \mathrm{~b}$, non-inferiority trial. Lancet 2017;389:2193-203.

14 Wykoff CC, Nittala MG, Zhou B, et al. Intravitreal aflibercept for retinal nonperfusion in proliferative diabetic retinopathy: outcomes from the randomized recovery trial. Ophthalmol Retina 2019;3:10761086.

15 Son G, Kim YJ, Sung YS, et al. Analysis of quantitative correlations between microaneurysm, ischaemic index and new vessels in ultrawide-field fluorescein angiography images using automated software. Br J Ophthalmol 2019;103:bjophthalmol-2018-313596.

16 Wessel MM, Aaker GD, Parlitsis G, et al. Ultra-wide-field angiography improves the detection and classification of diabetic retinopathy. Retina 2012;32:785-91.

17 Silva PS, Cavallerano JD, Haddad NMN, et al. Peripheral lesions identified on ultrawide field imaging predict increased risk of diabetic retinopathy progression over 4 years. Ophthalmology 2015;122:949-56.

18 Silva PS, Cavallerano JD, Sun JK, et al. Nonmydriatic ultrawide field retinal imaging compared with dilated standard 7 -field $35-\mathrm{mm}$ photography and retinal specialist examination for evaluation of diabetic retinopathy. Am J Ophthalmol 2012;154:549-59.

19 Bates DO. Vascular endothelial growth factors and vascular permeability. Cardiovasc Res 2010;87:262-71.

20 Fang M, Fan W, Shi Y, et al. Classification of regions of nonperfusion on ultra-widefield fluorescein angiography in patients with diabetic macular edema. Am J Ophthalmol 2019;206:74-81.

21 Bressler SB, Liu D, Glassman AR, et al. Change in diabetic retinopathy through 2 years: secondary analysis of a randomized clinical trial comparing aflibercept, bevacizumab, and ranibizumab. JAMA Ophthalmol 2017;135:558-68.

22 Dhoot DS, Baker K, Saroj N, et al. Baseline factors affecting changes in diabetic retinopathy severity scale score after intravitreal aflibercept or laser for diabetic macular edema. Ophthalmology 2018;125:51-6

23 Couturier A, Rey P-A, Erginay A, et al. Widefield OCT-angiography and fluorescein angiography assessments of nonperfusion in diabetic retinopathy and edema treated with anti-vascular endothelial growth factor. Ophthalmology 2019;126:1685-94. 